This item was submitted to Loughborough's Research Repository by the author.

Items in Figshare are protected by copyright, with all rights reserved, unless otherwise indicated.

\title{
A model of a repoint track switch for control
}

PLEASE CITE THE PUBLISHED VERSION

http://dx.doi.org/10.1109/CONTROL.2014.6915199

\section{PUBLISHER}

(c) IEEE

\section{VERSION}

AM (Accepted Manuscript)

\section{PUBLISHER STATEMENT}

This work is made available according to the conditions of the Creative Commons Attribution-NonCommercialNoDerivatives 4.0 International (CC BY-NC-ND 4.0) licence. Full details of this licence are available at: https://creativecommons.org/licenses/by-nc-nd/4.0/

\section{LICENCE}

CC BY-NC-ND 4.0

\section{REPOSITORY RECORD}

Wright, Nick, Samuel D. Bemment, Christopher P. Ward, and Roger Dixon. 2019. "A Model of a Repoint Track Switch for Control". figshare. https://hdl.handle.net/2134/17446. 


\title{
A Model of a Repoint Track Switch for Control
}

\author{
Nick Wright, Samuel Bemment, Christopher Ward, and Roger Dixon \\ Control Systems Research Group \\ School of Electrical, Electronic, \& Systems Engineering \\ Loughborough University \\ Leics, LE11 3TU \\ Corresponding Email: n.wright@lboro.ac.uk
}

\begin{abstract}
Track switching provides necessary flexibility to a rail network, allowing vehicles to change routes when necessary. Track switches, however, have historically been prone to failure. To increase asset reliability, a concept for a novel design of switch has been developed which allows multi-channel actuation through a novel actuation and locking mechanism, under a project titled 'Repoint'. This paper describes a mathematical model of the operation a novel Repoint track switch. The model was derived from a first principles physical analysis of the Repoint concept design. The structure of the model mimics the physical structure of the design. Each physical component has an individual submodel. The model has been used to estimate the actuator drive requirements for a case study mainline switch installation. It has been found that a Repoint track switch could be run from an existing UK signalling power supply. It is anticipated that this model will be used as the basis for a control system design activity for a technology demonstrator installation currently under construction.
\end{abstract}

\section{INTRODUCTION}

Track switching provides necessary flexibility to a rail network, allowing vehicles to change routes when necessary. However, track switches (sometimes referred to as points in the UK) represent single points of failure on a network. Even when operational, they represent a vehicular capacity constraint due to the associated systems of control and operation. Switches are expensive and complex designs when compared to equivalent plain line, as can be seen in industrial design and maintenance manuals [1], [2]. Their population is therefore generally optimised at design time alongside a known timetable in order to minimise initial outlay and substantial ongoing maintenance costs. This, however, has the effect of compounding the negative effects upon network performance during asset failures or other incidents which can perturb the normal running timetable.

Repoint [3] was a EPSRC and RSSB funded, 2-year project from 2011-2013, to explore the prospect of re-engineering railway track switches to embrace concepts from other industries, with the stated goal of demonstrating tangible capacity benefit from such an approach. These concepts principally included redundant, mission-critical subsystems, design for control, and in-built condition monitoring. The goal was to boost railway capacity by designing a switch which would allow the associated train control rules to be relaxed, due to its heightened intrinsic reliability and safety properties. One of the outputs of the project was an outline design for a switch which combined these principles, directly leading to two patent applications for novel aspects of the switch design (See [4], [5]), alongside a third for a novel crossing design.
The modelled capacity benefits of the Repoint design have been previously examined in [6], [7].

Improving maintainability and lowering asset lifecycle cost were not stated goals of the original project. However, the use of line-replaceable units and design for maintenance mean that the proposed concept design could achieve an in-service availability an order of magnitude greater than existing designs, alongside significant cost savings. In order to demonstrate the potential of the design, a laboratory scale concept demonstrator is currently at the design phase, and part of this design work is related to modelling the novel parallel actuator mechanism for the purposes of control system design, HIL simulation, and model-based condition monitoring.

Traditional switches come in various layouts, but generally consist of a single actuating element with an associated lock to hold the blades in position when set. This is coupled to electrical limit-switching to provide bang-bang control of the mechanism by simply cutting current to the actuator (or hydraulic power pack) once the blades are in the commanded position. There has been much recent study in modelling this layout, with the goal of retrofitting condition monitoring schemes to existing designs to improve reliability, for instance in [8]. However, it remains difficult to achieve the level of reliability desired by the industry.

The alternative approach is fault tolerance, that is, to detect and isolate a fault, whilst allowing the asset to continue to operate as required, perhaps through the use of subsystem redundancy, as discussed in [9]. The switch will have to be mechanically redesigned to some extent to allow this approach. A proposed novel layout, to this end, is shown in Fig. 1. Further design detail is available in [10].

The layout consists of a bank of parallel actuators, mounted inside the switch bearers, which bend the rail into each position through a semi-circular arc in the longitudinal plane. When the rail is in a lowered position, it rests inside grooves machined into the underside of each rail mounting. To enable a move between positions, the rail must first be raised before traversing and dropping. This allows the multiple actuation channels to work independently. Each actuator-bearer consists of a motor and sealed-unit gearbox, mounted in a position trackside (and therefore replaceable whilst the switch is in use). Actuatorbearers are independent of each other, only linked mechanically through the common rails, and electrically through the line-side control cabinet. Rails are mounted directly to the movable top of the bearers, referred to as the hopper, using standard rail mounts and clips. Within each bearer, motion is transferred from the gearbox output to the hopper through the 


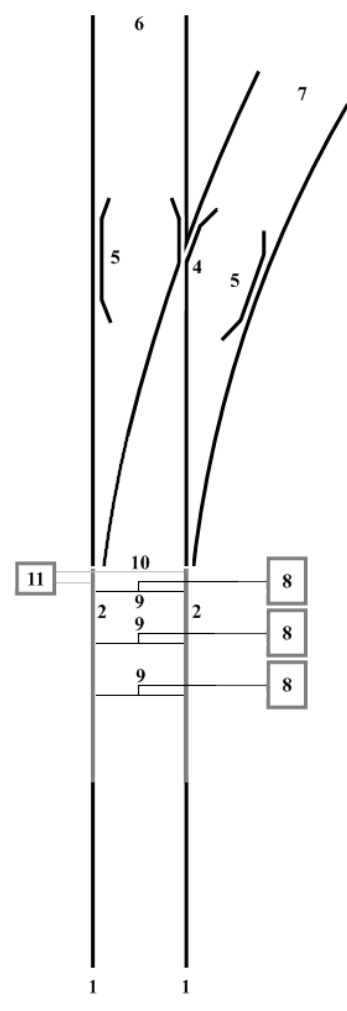

Fig. 1. Proposed REPOINT high-availability stub-switch concept with redundant actuation and locking paths. 1 (Black, Bold) Stock Rails; 2 (Grey, Bold) Moveable Switch Rails; 4 Common Crossing; 5 Check Rails; 6 Straight Route (herein, 'Normal' Route); 7 Turnout Route (herein, 'Reverse' Route); 8 Redundant Actuators, lineside type shown; 9 (Black) Drive Rod and Linkages ; 10 Detection Rods ; 11 Blade Position Detection and Feedback Unit Reproduced from [6]

use of an actuating rod with machined teeth on the underside, and twin idler cams moving in phase with each other. Figure 2 shows a schematic layout of a single actuator-bearer unit.

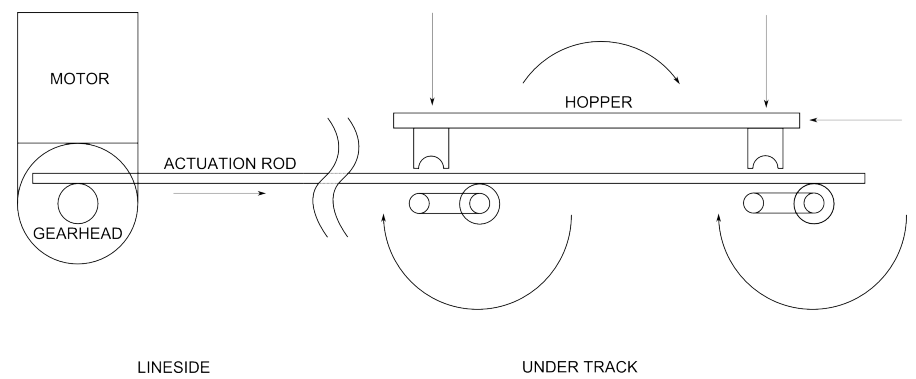

Fig. 2. Schematic representation of the mechanism inside each independent actuator-bearer unit. The Motor/gearhead are placed lineside in the end of the bearer with quick-release mounts. The switch rails mount to the upper surface of the hopper.

\section{The Repoint Model}

The Repoint switch model was derived from a first principles physical analysis of its component parts. The objective was to provide an easily accessible, transparent description of the physical behaviour of a switch. Different switch installations may have differing parameters, but the model and interaction of the subcomponents will remain the same. As such, the structure of the model mirrors the physical structure of the plant. The structure of the model is shown in Fig. 3. It has five main components: the brushless DC (BLDC) motor model, the gearhead model, the mechanical linkage model, the cam and hopper model, and finally, the rail pair model. Each of these components are discussed below.

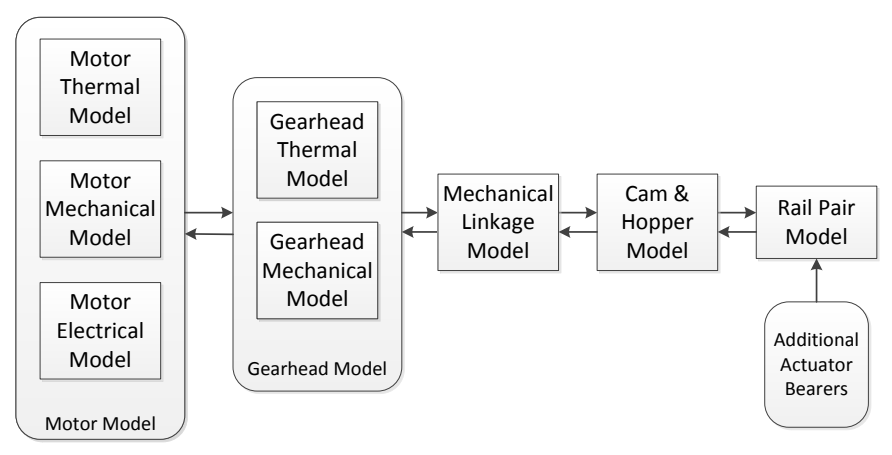

Fig. 3. An illustration of the Repoint model structure

\section{A. Motor Model}

BLDC motors are widely used devices and consequently have been well described in the literature. BLDC motors are polyphase devices that use electronic commutation to energise the correct motor armature winding according to speed and position in order to rotate the rotor. For the purpose of this model, however, it is assumed the electronic commutation is handled by the motor drive and all the motor phases are balanced. Real world DC motors will typically exhibit a degree of "torque ripple" caused by the commutation, but this was not deemed a significant factor for the control of a Repoint switch, and was therefore not included in the model. The main electrical, mechanical and thermal features of the motor are described by:

$$
V_{a}=R_{a} I_{a}+K_{v} \frac{d \theta}{d t}+L_{a} \frac{d I_{a}}{d t}
$$

Where $V_{a}$ is the motor terminal voltage, $R_{a}$ is the armature resistance, $I_{a}$ is the armature current, $K_{v}$ is the back emf constant, $L_{a}$ is the armature inductance, and $\theta$ is the angular position of the rotor.

The motor torque, $T_{m}$, is:

$$
T_{m}=I_{a} K_{t}
$$

$K_{t}$ is the motor torque constant. Therefore the mechanical output torque of the motor is described by:

$$
T_{m}-T_{g}=J_{m} \frac{d^{2} \theta}{d t^{2}}+B_{m} \frac{d \theta}{d t}
$$

Where $T_{g}$ is the gearhead torque, $J_{m}$ is the motor inertia, and $B_{m}$ is the motor friction constant.

The motor temperature, $\tau_{m}$, is described by:

$$
\frac{d \tau_{m}}{d t}=\frac{I_{a}^{2} R_{a}-K_{h} \tau_{m}}{C_{m}}
$$


Where $K_{h}$ is the motor head dissaption constant and $C_{m}$ is the heat capacity of the motor.

\section{B. Gearhead Model}

The main features of the gearhead that are of interest are the speed/torque ratio, the inertia, and the mechanical efficiency. It is envisioned that the Repoint design will use a high precision planetary gearhead, so for this particular component backlash is neglected, because it is expected to be small ( $<5$ arcmin).

The motor is connected to the gearhead by a short shaft, where the shaft output torque is given by:

$$
T_{s}=\left(\omega_{m}-\omega_{g i}\right) c_{s}+\int\left(\omega_{m}-\omega_{g}\right) k_{s}
$$

Where $T_{s}$ is the shaft output torque, $\omega_{g i}$ is the gearhead input speed, $c_{s}$ is the shaft damping constant, and $k_{s}$ is the shaft stiffness.

The output speed of the gearhead depends on both the assembly load torque and the shaft torque:

$$
T_{s}-\frac{T_{m a}}{n}=J_{g} \frac{d \omega_{g i}}{d t}+\frac{\omega_{g o}}{n} B_{g}
$$

And:

$$
\omega_{g i}=\frac{\omega_{g o}}{n}
$$

Where $T_{m a}$ is the mechanical assembly load torque, $\omega_{g o}$ is the gearhead output speed, $n$ is the gearing ratio, and $B_{g}$ is the gear damping constant.

The gearhead temperature is given by:

$$
\frac{d \tau_{g}}{d t}=\frac{T_{s} \omega_{g i}-T_{m a} \omega_{g o}-K_{g} \tau_{g}}{C_{g}}
$$

Where $\tau_{g}$ is the gearhead temperature, $K_{g}$ is the gearhead heat dissipation factor, and $C_{g}$ is the heat capacity of the gearhead.

\section{Mechanical Linkage Model}

The mechanical linkage is essentially a rack and pinion drive, connecting the motor gearhead to the two cams mounted underneath the hopper. The main features of this model section are the mass of the rack and the backlash between the pinion, cam gears, and rack. Unlike the gearhead model, backlash is considered here because experience suggests that over time it is possible that the rack may become displaced or deflected, increasing the effect of backlash. As such, it is useful to represent this in the model so that it can be accounted for in the control design. It is assumed there is a one to one gear ratio from the motor gearhead to the cam gears.

The equation of motion for the rack assembly is a discontinuous function, owing to the inclusion of backlash in this part of the model:

$$
f(x)= \begin{cases}0 & \text { for } \theta_{g}<\theta_{m} \\ \frac{T_{m a}}{R_{g}}-\frac{T_{h}}{R_{h}}=M_{m a} \frac{d^{2} x}{d t^{2}}+B_{m a} \frac{d x}{d t} & \text { for } \theta_{g} \geq \theta_{m}\end{cases}
$$

Where $x$ is the displacement of the rack, $R_{g}$ and $R_{h}$ are the gearhead and hopper pinion radius, $T_{h}$ is the load torque from the cam and hopper assembly, $B_{m a}$ is the rack assembly friction coefficient, $M_{m a}$ is the mass of the rack assembly, $\theta_{g}$ is the angular position of the gearhead, and $\theta_{m}$ is the angle at which the pinion engages with the rack.

\section{Cam and Hopper Model}

The mass of the hopper is balanced across two identical cams. It is anticipated that the greatest proportion of load on the cams will come from the elasticity of the rails, although the mass of the hopper itself will contribute. This is due to the fact the design bends the running rails in the vertical plane to move them between positions, and rails by their very nature are resistant to bending in this plane. For this iteration of the Repoint design, the profile of the cams was selected so that the follower (the hopper) moves through a half circle path. The torque from both cams is found by resolving the horizontal and vertical force components from the hopper:

$$
T_{h}=r_{c}\left(\cos \left(P_{v}+m_{h} g\right)+\sin P_{h}\right)+J_{h} \frac{d^{2} \theta_{c}}{d t^{2}}+\frac{d \theta_{c}}{d t} B_{h}
$$

Where $r_{c}$ is the radius of the cams, $P_{v}$ is the vertical force component on the hopper, $P_{h}$ is the horizontal force component on the hopper, $m_{h}$ is the mass of the hopper, $g$ is acceleration due to gravity, $J_{h}$ is the inertia of the cam and hopper, $\theta_{c}$ is the angular position of the cams, and $B_{h}$ is the friction coefficient between the cams and the hopper.

\section{E. Rail Pair Model}

As would be expected, the most significant load on each actuator bearer is the elasticity of the rail pair. To calculate the reaction force on each actuator bearer, the rail pair was treated as a lumped parameter, single beam. The beam deflection was calculated using McCaulay's method[11]. A shear diagram for the rail pair is shown below, in Fig. 4.

In Fig. $4, B_{A n}$ represent the weight of the fixings, $q$ is a uniform distributed self-load of the rail, $R_{A}$ is the vertical reaction at the anchor point, $x$ is the distance along the rail from the anchor point, and $P_{n}$ are the vertical forces from the actuator bearers. An equivalent diagram applies for the horizontal direction as well. Below, $E I$ is the beam bending stiffness (the product of its elastic modulus and area moment of inertia), $\theta$ is the angle of deflection, and $y$ is the magnitude of deflection.

Using McCaulays method for the shear force:

$$
\begin{aligned}
V(x)= & P_{1}<x-x_{1}>^{0}+P_{2}<x-x_{2}>^{0}+P_{3}<x-x_{3}>^{0} \\
& -q<x-x_{0}>^{1}-B_{A 1}<x-x_{1}>^{0} \\
& -B_{A 2}<x-x_{2}>^{0}-B_{A 3}<x-x_{3}>^{0}+R_{A}
\end{aligned}
$$




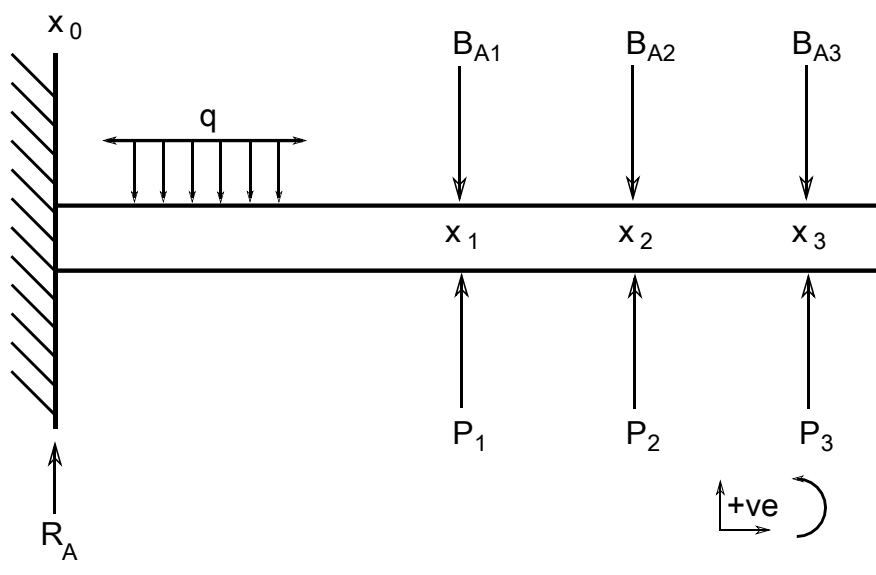

Fig. 4. A shear diagram for the rail pair model

For the bending moment:

$$
\begin{aligned}
M(x)= & P_{1}<x-x_{1}>^{1}+P_{2}<x-x_{2}>^{1}+P_{3}<x-x_{3}>^{1} \\
& -\frac{q}{2}<x-x_{0}>^{2}-B_{A 1}<x-x_{1}>^{1} \\
& -B_{A 2}<x-x_{2}>^{1}-B_{A 3}<x-x_{3}>^{1}+R_{A} x
\end{aligned}
$$

For the angle of deflection:

$$
\begin{aligned}
E I \theta & =\frac{P_{1}}{2}<x-x_{1}>^{2}+\frac{P_{2}}{2}<x-x_{2}>^{2}+\frac{P_{3}}{2}<x-x_{3}>^{2} \\
& -\frac{q}{6}<x-x_{0}>^{3}-\frac{B_{A 1}}{2}<x-x_{1}>^{2} \\
& -\frac{B_{A 2}}{2}<x-x_{2}>^{2}-\frac{B_{A 3}}{2}<x-x_{3}>^{2}+\frac{R_{A}}{2} x^{2} \\
& +C_{1}
\end{aligned}
$$

And for the magnitude of deflection:

$$
\begin{aligned}
E I y= & \frac{P_{1}}{6}<x-x_{1}>^{3}+\frac{P_{2}}{6}<x-x_{2}>^{3}+\frac{P_{3}}{6}<x-x_{3}>^{3} \\
& -\frac{q}{24}<x-x_{0}>^{4}-\frac{B_{A 1}}{6}<x-x_{1}>^{3} \\
& -\frac{B_{A 2}}{6}<x-x_{2}>^{3}-\frac{B_{A 3}}{6}<x-x_{3}>^{3}+\frac{R_{A}}{6} x^{3} \\
& +C_{1} x+C_{2}
\end{aligned}
$$

To find the constants:

For $C_{2}$, let $x=0, y=0$ therefore $C_{2}=0$.

For $C_{1}$, let $x=0, \theta=0$ therefore $C_{1}=0$.

\section{Simulation Results}

A simulation model of a prototype Repoint switch was complied using Matlab/Simulink [12]. The purpose of the simulation was to assist in the design of a lab-scale demonstrator and to investigate which components would be needed, for instance sizing of actuators and drive components. Two example configurations were investigated.

In the first, a switch has two exit routes, one perfectly straight on and another offset to one side, as in Fig. 1. In the second, the actuator bearer was aligned at the midpoint between the straight and turnout routes, effectively making the switch a 'Y-configuration'. In each case, it was assumed the cam had a radius of $80 \mathrm{~mm}$, and standard CEN60E1 flat bottom rail was used throughout. The distance of the actuator bearer from the anchor point - the movable length of rail was assumed to be $8 \mathrm{~m}$. This is the Repoint equivalent of a Type E switch, which is the most populous on the British network. The other two actuator bearers were assumed to be faulty and inoperable, to provide a "worst case" estimate of the cam torque that the bearer would be required to provide and the horizontal and vertical load on the bearer components.

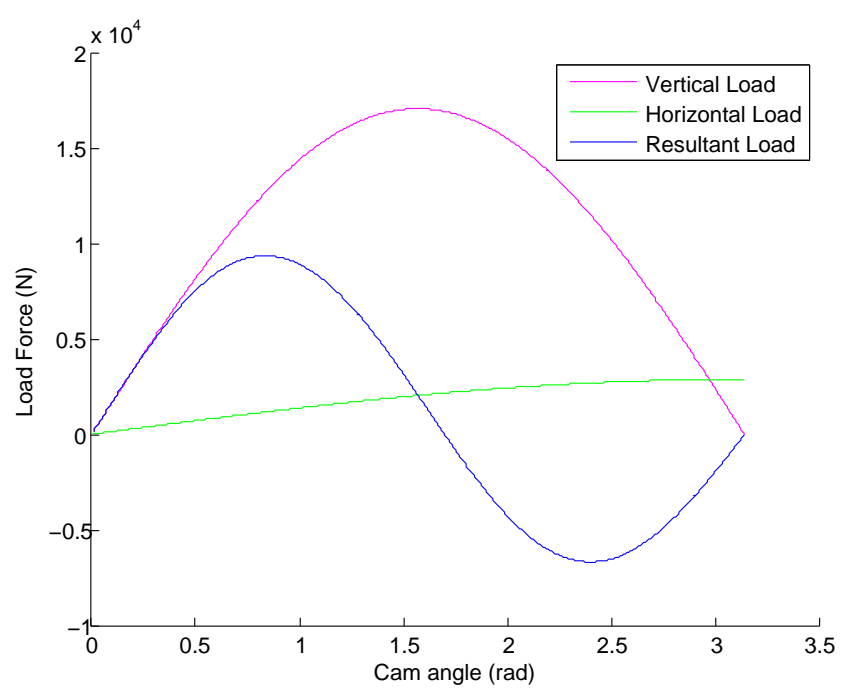

Fig. 5. Actuator bearer load, first configuration

Fig. 5 shows the estimated vertical and horizontal loads on the cams for switch movement in the first configuration. The resultant load on the cam in its direction of travel is also shown. It can be seen that halfway through the cam's motion, the resultant force works in the same direction as the cam.

Fig. 6 shows the torque on the cam. Again here it can be seen that in the second half of the motion range the cam itself is being driven by the elasticity of the rails.

The simulation results for the second configuration are shown in Fig. 7 and Fig. 8. The profile of the load and torque are similar, but their magnitude is around $20 \%$ smaller. This is due to the horizontal load working in the direction of motion of the cam during the first half of the motion cycle. The results suggest that the actuator requirements for an individual actuator bearer will show a significant degree of variability, according to the configuration of the switch and its layout. Care should be taken to design for the "worst case" design scenario.

Assuming a motor gearhead with a reduction ratio of 50:1 is used, the necessary torque output from the motor in 


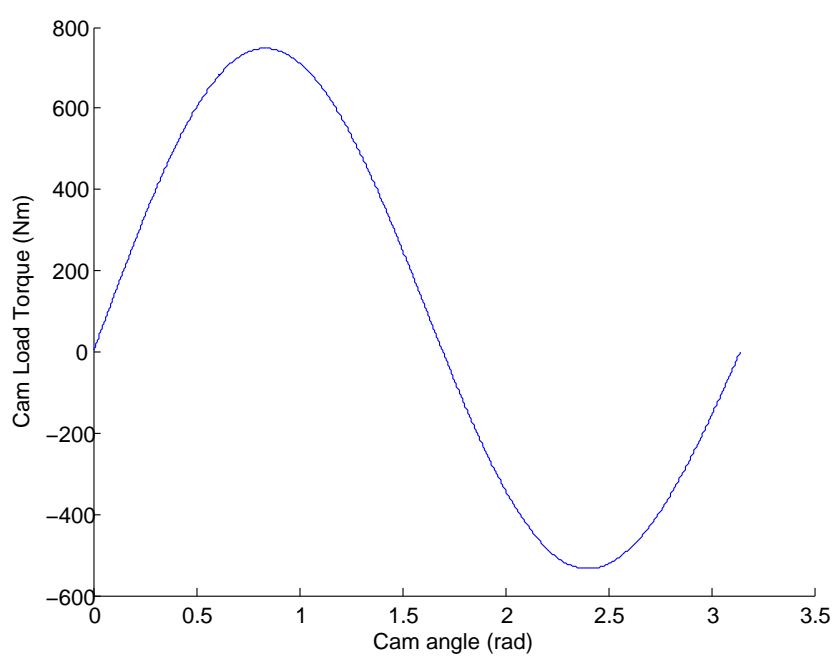

Fig. 6. Actuator bearer torque, first configuration

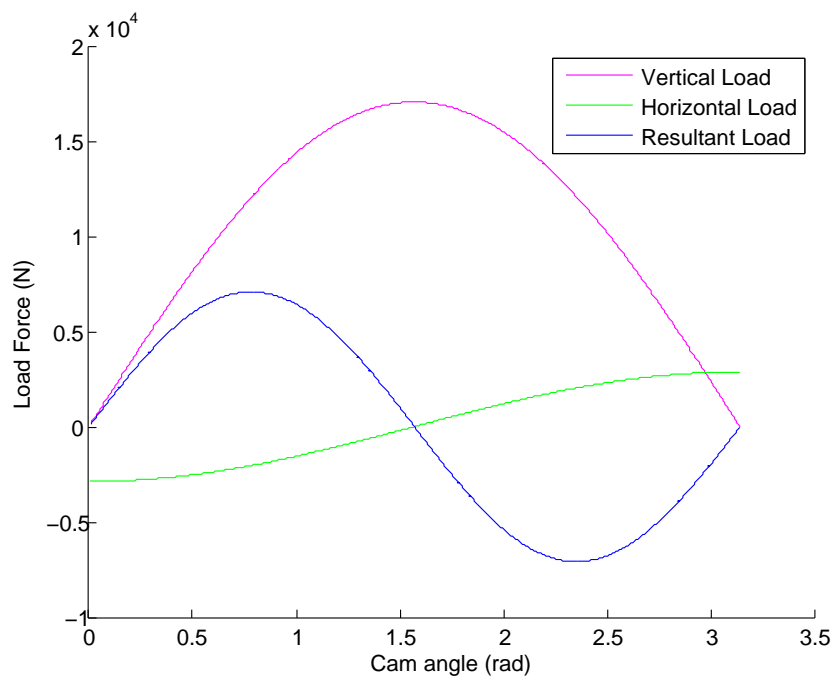

Fig. 7. Actuator bearer load, second configuration

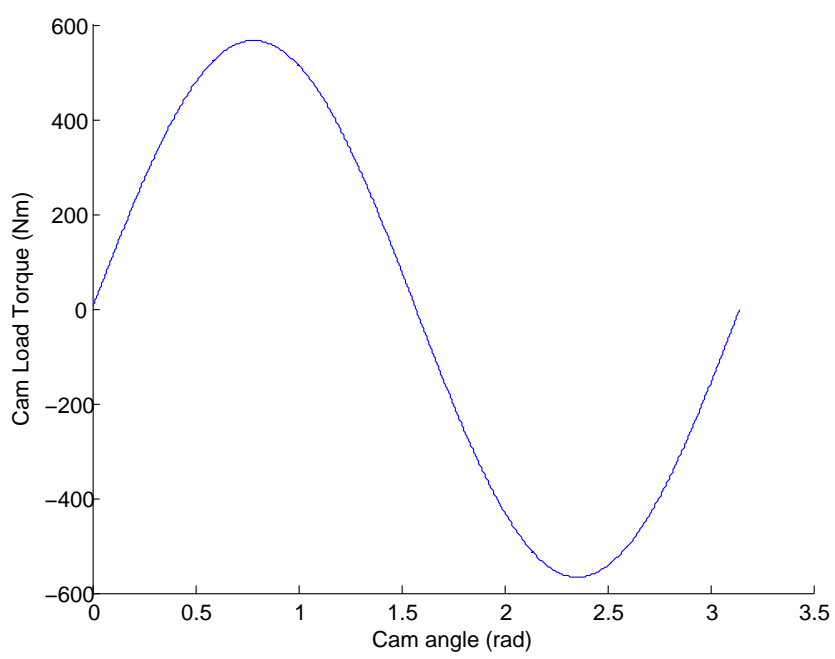

Fig. 8. Actuator bearer torque, second configuration the simulated actuator bearer is around $16 \mathrm{~N} / \mathrm{m}$ for the first (worst case) arrangement. Assuming the total actuation time has must similar to that of pre-existing UK switches (around 2 seconds[13]), then a wide range of suitable BLDC motors are available, a large selection of which could be powered by existing signalling power distribution units.

\section{CONCLUSION}

This paper has presented a simple mathematical model of a Repoint track switch. The model was derived from a first principles physical analysis of the design. The primary utility of this model lies in the fact it can be used as the basis for a control system design activity. It could also see secondary use in the selection of components and as the basis for a more detailed hardware-in-the-loop (HIL) simulation. When the model was implemented in Matlab/Simulink, it was estimated that the maximum torque that is required to operate the switch in the case of all but one actuator bearer failing would be around $800 \mathrm{~N} / \mathrm{m}$, based on the case examined.

The next phase of the Repoint project is to assemble a lab scale demonstrator of a single actuator bearer, to show that the design of a single bearer is practical. This phase of the project, along with the associated control system, is due to be completed in October 2014. This demonstrator will then be developed to use multiple parallel bearers, and the model presented herein will be used in the design of a control system to distribute load across those multi-channel actuator bearers.

\section{ACKNOWLEDGMENT}

The authors wish to acknowledge the financial support provided by the United Kingdom EPSRC (Engineering and Physical Sciences Research Council) and the UK Railway Safety and Standards Board RSSB in grant number EP/I010823/1, for the project titled 'REPOINT: Redundantly engineered points for enhanced reliability and capacity of railway track switching'. The authors also wish to acknowledge the support of the UK's EIT (Enabling Innovation Team), for providing followon research funding towards concept demonstrator construction (http://www.futurerailway.org/). The authors also wish to thank Tracsis PLC for ongoing industrial support and guidance.

\section{REFERENCES}

[1] D. D. L. Cope and J. B. Ellis, British Railway Track 7th Edition, Volume 5: Switch and Crossing Maintenance. Derby, UK: The Permanent Way Institution, 2002

[2] J. C. Morgan, British Railway Track 7th Edition, Volume 1, Design Part 2: Switches and Crossings. Derby, UK: The Permanent Way Institution, 2009.

[3] "EPSRC Grant Details: Redundantly Engineered POINTs (REPOINT) For Enhanced Reliability And Capacity Of Railway Track Switching," http://gow.epsrc.ac.uk/NGBOViewGrant.aspx?GrantRef=EP/I010823/1, accessed: 2013-10-12.

[4] Loughborough University, "Railway points operating apparatus (GB 1322660)," Patent GB 1322 660, 12 20, 2013.

[5] - "Railway points (GB 1322641)," Patent GB 1322641, 12 20, 2013.

[6] S. Bemment, R. Dixon, R. Goodall, and S. Brown, "Redundantly engineered track switching for enhanced railway nodal capacity," in Proc. 1st IFAC ACATTA Workshop, Istanbul, Turkey, September 2013. 
[7] S. Bemment, R. Dixon, and R. Goodall, "Repoint redundantly engineered points for enhanced reliability and capacity of railway track switching," in Proc. 10th World Congress on Railway Research (WCRR), Sydney, Australia, November 2013.

[8] J. Silmon and C. Roberts, "Improving switch reliability with innovative condition monitoring techniques." Proceedings of the IMechE, Part F: The Journal of Rail and Rapid Transit, vol. 224, pp. 293-302, 2010.

[9] R. Isermann, Fault-Diagnosis Systems: An Introduction from Fault Detection to Fault Tolerance. Berlin: Springer, 2006.

[10] S. Bemment, N. Wright, R. Dixon, C. Ward, and R. Goodall, "The performance and control requirements of a repoint track switch," in Proc. The Second International Conference on Railway Technology: Research, Development and Maintenance, Ajaccio, Corsica, France, April 2013.

[11] W. Macaulay, "Note on the deflection of beams," Messenger of Mathematics, 1919.

[12] Matlworks Inc., "MATLAB Documentation (Online)," 2013. [Online]. Available: http://www.mathworks.co.uk/help/matlab/

[13] J. F. H. Tyler, British Railway Signalling Practice - Signalling Relays And Circuits No. 6. Reading: Institution of Railway Signal Engineers, 2005 